\title{
Mass Gathering (Hajj) Pharmacy Infection Control: New Initiative in Saudi Arabia
}

Yousef Ahmed Alomi* iD, Critical Care Clinical Pharmacists, TPN Clinical Pharmacist, Freelancer Business Planner, Content Editor and Data Analyst, Riyadh, Saudi Arabia.

Lamees Essa Abdullah Hakami, Pharm D

College of Pharmacy, Jazan University, Jazan Region, Saudi Arabia.

Nezar Adnan Yahya Khayat, Bsc. Pharm Medication Safety Officer, Pharmaceutical Care Administration, Makkah Medical Affairs Region, Makkah, Saudi Arabia.

Yasir Abdulraheem Bamagaus, Bsc. Pharm Regional General Manager, Pharmaceutical Care Administration, Makkah Medical Affairs Region, Makkah, Saudi Arabia.

Taha Mohamd Rafuden Bakhsh, Bsc. Pharm Staff Pharmacist, Pharmaceutical Care Administration, Makkah Medical Affairs Region, Makkah, Saudi Arabia.

Nojoud Adnan Khayat, Bsc. Pharm

Staff Pharmacist, Pharmaceutical Care Administration, Makkah Medical Affairs Region, Makkah, Saudi Arabia.

Ahmad M. AlKharoby, MD, Assistant General Manager for assistant medical affiars, Makkah Medical Affairs Region, Makkah, Saudi Arabia. Wael H. Motair, MD, General Manager, Makkah Medical Affairs Region, Makkah, Saudi Arabia.

\section{Correspondence:}

Dr. Yousef Ahmed Alomi, (BSc. Pharm, MSc. Clin Pharm, BCPS, BCNSP, DiBA, CDE)

Critical Care Clinical Pharmacists, TPN Clinical Pharmacist, Freelancer Business Planner, Content Editor and Data Analyst, P.O. BOX 100,

Riyadh-11392, Riyadh, Saudi Arabia.

Phone no: +966504417712

E-mail:yalomi@gmail.com

Received: 13-08-2019;

Accepted: 25-11-2019

Copyright: (c) the author(s), publisher and licensee International Journal of Pharmacology and Clinical Sciences. This is an openaccess article distributed under the terms of the Creative Commons Attribution NonCommercial License, which permits unrestricted non-commercial use, distribution, and reproduction in any medium, provided the original work is properly cited.

This is an open access article distributed under the terms of the Creative Commons Attribution-NonCommercial-ShareAlike 4.0 License

Access this article online

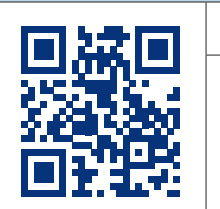

WwW.ijpcs.net

DOI:

10.5530/ijpcs.2020.9.2

\begin{abstract}
Objectives: To demonstrate the pharmacy infection control services during the mass gathering Hajj period as new initiatives in the Kingdom of Saudi Arabia. Methods: It is a new initiative project drove by national mass gathering pharmaceutical programs. The projects drove the mass gathering medicine guidelines and the international business model, pharmacy project guidelines project management institution guidelines of a new project. The initiative project is written through project management professionals and consisted of several parts, including the initial phase, the planning phase, the execution phase and the monitoring and controlling phase. Results: The mass gathering pharmacy infection control services with a defined vision, mission and goals. The services had various aids, including clinical and economical on pilgrims, as illustrated in the assessment. The continuation was of the project assured by the risk management model description. Besides, the monitoring and controlling of the services as declared. The transition to operation project, though closing the project stage, explored in the analysis. Conclusion: The mass gathering pharmacy infection control services are a new initiative, part of the mass gathering medicine. There are various pharmacy transition care services can be started during the mass gathering with an emphasis on pharmacy personnel, infection control; it is highly suggested to implement in Saudi Arabia.
\end{abstract}

Keywords: Mass gathering, Hajj, Infection, Control, Pharmacy, Services, Initiative, Saudi Arabia.

\section{INTRODUCTION}

Saudi Arabia obtains every year, a large number of pilgrims of more than 2 million pilgrims from all over the world. ${ }^{[1]}$ Hajj is compulsory on every Muslim and is the fifth pillar of Islam. The Hajj has performed annually in the city of Mecca. Considered as the largest gathering in the world. This association may occur with a massive outbreak of infectious diseases that affect pilgrims and may be transmitted to the local population, where the number of pilgrims this year will be more than the last years. ${ }^{[2-5]}$ Saudi newspaper reported that the number of outside pilgrims this year increased by 5\%,12\% and 7\% through air, land, sea traveling respectively, compared to last year. ${ }^{[6]}$ The rate of transmission of communicable diseases among pilgrims is very high, as congestion and the proximity of pilgrims to each other is a critical factor in the transmission of infection, the inhalation of polluted air, the use of personal objects, sunstroke and others. ${ }^{[7,8]}$ Saudi Arabia is taking measures to protect pilgrims and their safety. The government also provides umbrellas and fans with water spray to soften the atmosphere and protect them from harmful sun rays, where many pilgrims suffer from sunstroke. Where Saudi Arabia is working on strategies and surveillance systems, including implementation of the Infectious Diseases Surveillance System (IDSS) to report disease outbreaks to the responsible authorities and the government recognized the command and control center (CCC) to detect middle east respiratory syndrome coronavirus
(MERS-CoV) outbreaks starting from 2012 until now. ${ }^{[9]}$ In this project, infectious diseases that can be transmitted between pilgrims during the Hajj season and how to prevent and control them by the pharmacist and what are the necessary precautions before and during the mass gathering Hajj. The role of the pharmacist is vital in infection control. Several studies discussed pharmacy infection control as general. However, the mass gathering pharmacy infection control based on basic knowledge of authors, not published locally or in the Gulf or Middle East countries or the rest of the world. The aim of this topic is to declare the project review about mass gathering pharmacy information control in the Kingdom of Saudi Arabia.

\section{Most Infectious Disease during Hajj}

Multiple diseases might find during the mass gathering hajj period; for an instant, Respiratory infections, which were respiratory inflammation is one of the most contagious diseases during Hajj, which was first discovered in Hajj in 2012. [10] Congestion may be a cause of pneumonia disease. ${ }^{[1]}$ There are many types of viruses that infect the respiratory system during the pilgrimage season and most common East respiratory syndrome coronavirus (MERS-CoV) ${ }^{[11,12]}$ and influenza A H1N1. ${ }^{[11,13]}$ The Meningococcal disease or meningitis, which are a common infectious disease during the Hajj pilgrimage. The first appeared of disease in Hajj was in 1987 and 
outbreak in 2000-2001, where the disease is transmitted through interpersonal communication through the oropharyngeal secretions. ${ }^{[14,15]}$ Neisseria meningitides serogroup W135 (W135) were reported in the Hajj..$^{[14-16]}$ The Tuberculosis disease might found with overcrowded may be a factor in the transmission of tuberculosis among the pilgrims. Tuberculosis is one of the infectious diseases transmitted in large gatherings such as Hajj. The physician recommends immediate examination when symptoms such as a persistent cough or blood appear during cough and general weakness of the body. ${ }^{[10]}$

\section{How to Control Infectious Disease during the Mass Gathering Hajj Period}

In Respiratory infections; Studies have shown that face mask is effective in protecting against flu and the spread of diseases transmitted by coughing and sneezing. ${ }^{[10,11,17]}$ The Recommendations making a diagnosis to identify the etiology of the disease. ${ }^{[11,18]}$ The vaccination limits the transmission of communicable diseases among pilgrims such as pneumococcal disease, which may be lethal, as reported in 35\% fatal case. Pneumococcal polysaccharide vaccine suggested for all, especially elderly people and those at risk, Seasonal influenza vaccine, including $\mathrm{H} 1 \mathrm{~N} 1$, is strongly recommended for all pilgrims. ${ }^{[10,18]}$ Influenza vaccination to prevent and reduce the frequency outbreak of disease. ${ }^{[11]}$ While Meningococcal disease or Meningitis, which is a medical emergency and Saudi Arabia is working hard to limit the outbreak of the disease among pilgrims. ${ }^{[14]}$ Take an antibiotic as chemoprophylaxis is essential to reduce the risk of meningococcal disease among pilgrims and there are more than one of antibiotics can treat the infectious disease as oral ciprofloxacin, penicillin, ampicillin as well as ceftriaxone and ceftriaxone is a choice drug in Africa. Saudi Arabia ordered pilgrims aged two and above to be vaccinated against meningitis at least ten days before going to the Hajj where pilgrims receive (ACYW135) meningococcal vaccine, Polysaccharide vaccine administration by subcutaneous and Protein-based vaccine. ${ }^{[9,10]}$ Also, The Tuberculosis disease needs to apply a face mask and replace it every time to prevent the spread of viruses transmitted by coughing or sneezing, hand wash and maintain overall cleanliness of the body, stay away from very crowded locations as much as possible and stay in proper ventilation. ${ }^{[19]}$ Pilgrims can get on the vaccine as prophylaxis before going to hajj as bacilli Calmette-Guérin (BCG). ${ }^{[20]}$

Besides, other ways may be missed by pilgrims subsidize to the transmission of diseases from one patient to another, including the use of the same scissors or shaving the head with the same shaving machine or without changing the razor blade, the use of personal determinations such as comb, nail clippers, soap and others. The pilgrim must protect himself first by preserving his personal items and not sharing them with other people and that all his purposes should be complete so as not to use the purposes of others and not use the same face mask from person to person. Bring a new shaving machine and a new razor blade to protect against hepatitis $A$ and $B$ as well as protect from AIDS. These approaches help lessen the risk of transmission of infectious diseases. ${ }^{[21]}$ With overcrowding, infectious diseases abound, especially in large gatherings such as the Hajj, which contains more than two million pilgrims. Therefore, a human needs to protect them from transmitted diseases, so it is essential to take precautions and the necessary measures and the most important preventive measures are vaccinated. Vaccination is a necessary preventive measure of diseases, where the ministry of health orders to take vaccination against meningitis, which affects the brain membranes and suggested pilgrims take vaccination against seasonal flu as it is a common disease in Hajj that harm the health of the pilgrim. Vaccination has contributed to a lessening in morbidity and mortality. [21]

\section{Method of the Project}

It is a new initiative project drove by national mass gathering pharmaceutical programs. The task force team of mass gathering hajj pharmacy projects formulated and consisted of from an author's expert in the mass gathering hajj. The committee unitized and drove the mass gathering medicine guidelines and from the textbook and international literature about mass gathering medications, safety written by utilizing the international business model, pharmacy project guidelines project management institution guidelines of a new project. [22-25] The mass gathering medication safety project written through project management professionals and contained several parts, including the initial phase, the planning phase, the execution phase and the monitoring and controlling phase.

\section{Initial Phase}

\section{Assessment Needs}

The pharmacist has a vital role in the clinical practice, pivotal role in infection prevention as well as an active role in the pilgrimage season, including Providing pharmaceutical care for pilgrims, correcting misconceptions about the uses of medicines in general and antibiotics in particular so as not to occur resistance to bacteria, also has a vital role in providing informa- tion to the medical team and patients. To know the role of the pharmacist in the pilgrimage season, it is required to appoint pharmacists with involvement on infectious diseases in the pilgrimage season and pharmacists for health education from the poison information center and others and allow them to participate in determining the pharmacist's role in the pilgrimage.

\section{Market Analysis}

Several pharmacy practice programs started several years back, for instance, the antimicrobial stewardship program. Several healthcare organizations implemented these projects. The elements of infection control had been implemented at most healthcare organizations with governmental or private sectors. However, the implementation of infection control in pharmacy services, not entirely excised. As a result, pharmacy infection control and prevention are essential to project to prevent pharmacy outbreak infection and infectious disease transmission throughout the pharmacy departments. The crowded area, like mass gathering hajj, is highly required for precautionary infection control. The pharmacy infection control during the mass gathering hajj period highly demands in the Kingdom of Saudi Arabia.

\section{SWOT Analysis}

In this project, we performed a SWOT analysis of the pharmacy role in infection control in the Hajj season. The strength of the project gives pharmacists a great occasion to contribute in the Hajj season, prove the role of the pharmacist in infection prevention and in society through the documentation. The weakness of the project was the lack of time to make documents for the role of the pharmacists in the infection control because the pilgrimage season is once a year and in 7-8 days, a large number of pilgrims and the busy medical team to work documents. The chances were fitting for pharmacists to prove their role in the pilgrimage season and fitting for medical care in order to provide medical services to pilgrims such as infection control. The threats of the project are poor knowledge of the pharmacist's role in Hajj and infection control due to lack of time to document the role of the pharmacist in infection control dur-ing the Hajj season.

\section{The Scope of the Project}

The system pharmacy infection control and prevention that's including the pharmacist performance in infection deterrence within the pharmacy services during the mass gathering hajj period. The proper choice of antibiotics and vaccination, pharmacist role in educating the medical team about the medication and 
patient counseling related to infection control. It also includes the clinical pharmacists' interventions offered to the patient, their type, method of treatment and type of treatment, potentials and outcomes related to pharmacy infection control.

\section{Vision, Mission and Goals}

The vision of the project is to achieve the Saudi Arabia vision 2030 in clinical practice and to change the concept the pharmacist role in the Hajj season in infection control. The mission of this project is to progress, employment and monitoring of antimicrobial resistance and vaccination to control of infectious disease in Hajj and innovate pharmacy infection control related issues. The goals of this project is implemented infection control in the pharmacy practice during the mass gathering hajj period, to prevent infection control related problems and to provide prospects for pharmacists to work in more than one area, to prove the vital role of the pharmacist in the medical team during the pilgrimage season of infectious disease prevention in the public.

\section{Project Description}

The pharmacists and other health care professional should know the policies, procedure and precaution for infection prevention and control (IP and C) practice that given below in the pharmacy practice as general with emphasis on the mass gathering hajj:

- Hand washes after and before contact with patients after the intervention and after use, anything can cause contamination. Hand wash with soap frequently and by right way and using an antimicrobial soap, antiseptic agent and can use sterile, contain at least $60 \%$ of alcohol as well as sterile tissue as an alternative way.

- Wear face masks to protect him/herself from any infection that transfer by air, wear gloves to protect from bacteria by contact and an eyeglass.

- Take vaccines against the infectious disease before traveling to Hajj.

- If health care professionals have any disease that should do not work, if it is necessary to work should maintain appropriate space with patients and wear a face mask.

- Pharmacists and other health care professionals should be known how to achieve adequate infection control of education and training.

- Have appropriate immunization with a valid date to use, for example, vaccine available for the most common infectious disease as meningococcal disease, respiratory infection and tuberculosis.
- Use appropriate antibiotics for bacterial infections like Clostridium difficile. For example, penicillin, cephalosporin, vancomycin, aminoglycoside and other essential antibiotics.

- The pharmacy room in Hajj should be apparent daily and keep the pharmacy ventilation well to protect from contamination and protect medications by removing the dust, west, cartons and bottles and should contain dedicated handwashing, alcohol hand rub and eyewash for the emergency station.

- The pharmacist is accountable for the training of patients and other health, medical care about: hand washes, workplace hazardous materials information system (WHMIS), vaccine and medication management and storage.

- Pharmacists and other staff should obtain training about clinical practice guideline infection control and prevention related to infectious diseases.

- Pharmacy infection control contained the following aspects:

$\checkmark$ Pharmacy service: The patient that stays in the hospital the whole day or more than a day. This area contribution to education and training the medical team and make counseling with patients and their families present in the hospital at the bed about antimicrobial use, dose, duration, administration and other medication that uses in infectious disease and another disease. The area needs to clear, sterile and should put alcohol sterilizer in all departments and ward to prevent the speared of bacteria and other microorganisms. For example, Isopropyl alcohol 70\% (IPA) is the most common use because it is quickly antimicrobial against bacteria, fungi and viruses and less time use than water with soap. Its uses after anything make contamination of hand and before a meeting of the patient.

$\checkmark$ Outpatient pharmacy: Pharmacy service to the patient who does stay in the hospital the whole day. In this area, do the same service to the patient like inpatient, but the pharmacist does not need to go towards he/she can make counseling with the patient at the pharmacy window and also need to be sterile to evade infectious disease.

$\checkmark$ IV admixture: A preparation of two or more of medication in a bottle of IV fluid, it does by a pharmacy technician and check with a pharmacist or clinical pharmacy. The syringe should retain in packaging to protect from contami- nation and need to change the syringe at every use for every patient and discarded in a sharp's container, clear the room every day by alcohol sterilizer, wear surgical gowns and face mask before preparation, do not eat or drink or perform in the IV room.

$\checkmark$ Extemporaneous preparation unit: Extemporaneous compound in the preparation of a drug for an individual patient to a specific purpose; when the drug or dosage form not available and dispensing instantly after preparation such as oral product, eyes, ear or nasal drops and parenteral injection should prepare under aseptic technics.

$\checkmark$ The clinical pharmacy unit is contributing to work directly with physicians, another medical team and patients with education, training and counseling. Clinical pharmacy contributes topatient care, provides better patient health outcomes and in making the discussion about good medication therapy for the patient and should personal defensive measures.

$\checkmark$ Medication safety and pharmacy quality management are units support the excellent and safety use of drug by providing accurate information about medication or solutions used for infection control, correct informed and used error of medication and provide information about poisons and cytotoxic medication, drugs interaction, food and drug interaction, allergy from medicine, dose, duration and administration of medication and high awareness of people about correct use of antibiotics and other medication to ensure the quality management and medication safety to save the patient's life.

$\checkmark$ The pharmacy store should contain all medication that needs in the management of infectious disease, chronic disease, injuries and other common diseases in hajj and easy access to medication. Keep pharmacy store clean with cleaning the floor and shelves every day by isopropyl alcohol at less two times in the day, disposal of waste immediately, supply the pharmacy store with ventilation well and refrigerator with a temperature between $2^{\circ} \mathrm{C}-8^{\circ} \mathrm{C}$. The store should be protected from sunlight.

$\checkmark$ Immunization service for pharmacy staff: Staff who is dealing with patients and infection disease should receive a successful immunization against infection such as hepatitis B, meningococcal, influenza and pneumococcal polysaccharide vaccine to prevent the spread of 
disease.

$\checkmark$ Immunization for accidental procedures: Vaccine averts many diseases as well as the accident that occurred from the wrong procedure such as blood infection and HIV sometimes occur from the contaminated needle use or wrong cutting procedure. To stop this from occurring first should be cautious about dealing with needles and cutting procedures, training for the successful procedure before work on a patient, disposal of contaminated needle and knives, wear gloves and glass before doing any procedure. Immunization had an essential role in preventing this infection, such as a give MMR vaccine for HIV deterrence and can give to children because it is safe.

$\checkmark$ Pharmacy infection control surveillance: Pharmacists have a role in reducing infection by surveillance of microorganisms to improve antimicrobial use to reduce drug resistance, a side effect of drugs and monitor other interactions, such as allergy, drug-drug interaction and drug-food interaction to ensure optimal pharmacotherapy.

\section{Cost Management Plan}

The financial budget is one of the crucial things to implement the mass gathering hajj pharmacy infection control. The budget consisted of multiple sections that included but not limited to education and training of mass gathering hajj pharmacy infection control, the salary of all pharmacy staff working as pharmacists and pharmacy technician infection control, computerized system for data enters of pharmacy infection control and the intensives of management team meetings. The defined budget should monitor throughout the project through the implementation processes.

\section{Execution Phase \\ Management Team}

The pharmacist infection control team should establish the mass gathering hajj pharmacy infection control in addition to contained of clinical pharmacists and expert pharmacists and pharmacy technicians and pharmacy total quality management. The team should implement the project by the education and training the pharmacists and pharmacy technicians in pharmacy infection control during the mass gathering hajj. The team should organize the policy and procedures of the project and monitoring the employment of the program and measure clinical with the economic outcome of the new project.

\section{Education and Training}

The pharmacy infection control needs several courses for all pharmacy staff, including clinical pharmacists, pharmacists and pharmacy technicians, about the updated communicable disease management, immunization therapy and personal protective measure. On other hand, other part education is a material solution for infection control and immunization for healthcare providers and orientation lectures for all physicians and nurses. Some educational courses for the management team, including the management team and higher administration, moreover, about clinical and economic outcomes of pharmacy infection control.

\section{Monitoring and Controlling Phase Project Quality Management}

The recent new model for total quality measurements of the new project is Balance Scored Card and it cans used for mass gathering hajj pharmaceutical care. The BSC contained four elements of measure that's including the customer, finance, internal process, education and innovation. The explored example of internal processes was the assessment of mass gathering hajj pharmaceutical care in practice. The type the education and innovation were the measures of clinical outcome of a mass gathering of pharmaceutical care that's declared the education and competency of pharmacy staff during perform the project. Next element example related to the financial type the measurement of the economic impact of mass gathering hajj pharmaceutical care services. While the customer types may measure the patients, pharmacy, pharmacists and pharmacy technicians and healthcare providers about mass gathering hajj activity satisfaction in Saudi Arabia.

\section{Risk Management}

Various types of risk management include budget risk, scope risk, personal risk, schedule risk, technical risk and quality risk. The project might be exposed to risks such as personnel, budget, technical and quality risks. The project adequately exposed to personal risk with a shortage of pharmacy staff during the mass gathering hajj period. The budget risk might be exposed to the current project with a shortage of financial budget related team management members or for education and training. Another type of risk called the technical risk and the solution for infection control not available. The mass gathering hajj pharmacy infection control may be exposed to the quality risks due to the non-qualified or expert pharmacist being available during the mass gathering hajj period.

\section{The Closing of the Project}

The mass gathering of pharmacy infection control at all healthcare institutions, both governmental and private, is highly suggested to pharmacy-related infection that might lead to morbidity. Moreover, to prevent the economic burden on the healthcare system at hospitals and primary healthcare centers in Saudi Arabia. The project should include an extension of all pharmacy services and related committees. The mass gathering pharmacy infection control Education and training should be done regularly. Update the pharmacy infection control with new models and antiseptic and other materials are necessary for the future. The Annual celebration of mass gathering pharmacy infection control of all staff, including clinical pharmacists, pharmacists and pharmacy technician's at all healthcare institutions are highly recommended in Saudi Arabia.

\section{ACKNOWLEDGEMENT}

None.

\section{CONFLICT OF INTEREST}

The authors declare no conflict of interest.

\section{ABBREVIATIONS}

IDSS: Infectious disease surveillance system; CCC: Command and control center; MERS CoV: Respiratory syndrome coronavirus; ASP: Antimicrobial stewardship program; BCG: bacilli Calmette-Guérin; CPA: Commonwealth pharmacists association; PROMISe: The Pharmacy Recording of Medication Incidents and Services electronically; IPA: Isopropyl alcohol 70\%; MOH: Ministry of Health; SWOT: Strength, Weakness, Opportunities, and Threats; WHMIS: Workplace Hazardous Materials Information System; ASHP: Healthsystem Pharmacists; MMR: Measles Mumps Rubella; HIV: Human Immunodeficiency Virus.

\section{REFERENCES}

1. Alomi YA, Alghamdi SJ, Alattyh RA. National survey of pharmacy practice at $\mathrm{MOH}$ hospitals in Saudi Arabia 2016-2017: Clinical pharmacy services. J Pharm Pr Community Med. 2018;4(1):1S-8S.

2. Memish ZA, Zumla A, Alhakeem RF, et al. Hajj: Infectious disease surveillance and control. Lancet. 2014;383(9934):2073-82.

3. Ebrahim SH, Doumbia S, Elachola H, et al. Healthy Hajj 2019 - what you need to know, before you go. Travel Med Infect Dis. 2019;40:2018-20.

4. Shafi S, Dar O, Khan M, et al. The annual Hajj pilgrimage-minimizing the risk of ill health in pilgrims from Europe and opportunity for driving the best prevention and health promotion guidelines. Int J Infect Dis. 2016;47:79-82.

5. Gautret P, Steffen R. Communicable diseases as health risks at mass gathering other than Hajj: What is the evidence?. Int J Infect Dis. 2016;47:46-52. 
6. General Authority for Statistics. Hajj Statistical Book in Kingdom of Saudi Arabia. 2019.

7. Alqahtani AS, Althimiri NA, BinDhim NF. Saudi Hajj pilgrims' preparation and uptake of health preventive measures during Hajj 2017. J Infect Public Health. 2019;5-9.

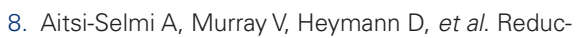
ing risks to health and wellbeing at mass gathering: The role of the sendai framework for disaster risk reduction. Int J Infect Dis. 2016;47:101-4.

9. Alotaibi BM, Yezli S, BinS AAA, et al. Strengthening health security at the Hajj mass gathering: Characteristics of the infectious diseases surveillance systems operational during the 2015 Hajj. Journal of Travel Medicine. 2017;24(3)

10. Saad A. Health issues during Hajj. Egypt $J$ Intern Med. 2017;29(2):37.

11. Al-Tawfiq JA, Gautret P, Benkouiten S, et al. Mass gathering and the spread of respiratory infections lessons from the Hajj. Ann Am Thorac Soc. 2016;13(6):759-65.

12. Memish ZA, Almasri M, Turkestani A, et al. Etiology of severe community-acquired pneumonia during the 2013 Haji-part of the MERS-CoV surveillance program. Int J Infect Dis. 2014;25:186-90.
13. Memish ZA, McNabb SJN, Mahoney F, et al. Establishment of public health security in Saudi Arabia for the 2009 Hajj in response to pandemic influenza A H1N1. Lancet. 2009;374(9703):1786-91.

14. Yezli S, Assiri AM, Alhakeem RF, et al. Meningococcal disease during the Hajj and Umrah mass gathering. Int J Infect Dis. 2016;47:60-4.

15. Wilder-Smith A, Goh KT, Barkham T, et al. Hajj-associated outbreak strain of Neisseria meningitidis serogroup w135: Estimates of the attack rate in a defined population and the risk of invasive disease developing in carriers. Clin Infect Dis. 2003;36(6):679-83.

16. Zhujun SHZ, Yuan G, Hongyu R, et al. Neisseria meningitidis Serogroup W135, China. J Infect Dis. 2002;185(11):1596-605.

17. Barasheed O, Alfelali M, Mushta S, et al. Uptake and effectiveness of face mask against respiratory infections at mass gathering: A systematic review. Int J Infect Dis. 2016;47:105-11.

18. Al-Barrak A, Alotaibi B, Yassin $Y$, et al. Proportion of adult community-acquired pneumonia cases attributable to Streptococcus pneumoniae among Hajj pilgrims in 2016. Int J Infect Dis. 2018;69:6874
19. Kaufmann SHE, Weiner J, Reyn CFV. Novel approaches to tuberculosis vaccine development. Int J Infect Dis. 2017;56:263-7.

20. Memish ZA. The hajj: Communicable and noncommunicable health hazards and current guidance for pilgrims. Eurosurveillance. 2010;15(39):14.

21. Abd El Ghany M, Sharaf H, Hill-Cawthorne GA Hajj vaccinations-facts, challenges and hope. Int J Infect Dis. 2016:47:29-37.

22. McDonough R. Writing a Business Plan for a New Pharmacy Service. The Dynamics of Pharmaceutical Care: Enriching Patients' Health. 2010;23.

23. Harris IM, Baker E, Berry TM, et al. Developing a Business-Practice Model for Pharmacy Services in Ambulatory Settings. Pharmacotherapy. 2008:28(2):7e-34e.

24. Sachdev G. Sustainable business models: Systematic approach toward successful ambulatory care pharmacy practice. Am J Heal Pharm. 2014;71(16):1366-74.

25. PMBOK Guide. A guide to the project management body of knowledge. Sixth Edition. Project Management Institute, Inc. 2017. 